OPEN ACCESS

Edited by: Onder Alpdogan, Thomas Jefferson University, United States

Reviewed by: Anne Reiner,

Memorial Sloan Kettering

Cancer Center, United States Gaurav Prakash

Post Graduate Institute of Medical

Education and Research (PGIMER), India

${ }^{*}$ Correspondence: Leyuan Wang happygarden01@csu.edu.cn

Specialty section: This article was submitted to Hematologic Malignancies, a section of the journal

Frontiers in Oncology

Received: 18 October 2020 Accepted: 05 March 2021

Published: 26 March 2021

Citation:

Feng Y, Liu Y, Zhong M and Wang $L$ (2021) Complete Blood Count Score

Model Predicts Inferior Prognosis in

Primary Central Nervous System Lymphoma.

Front. Oncol. 11:618694. doi: 10.3389/fonc.2021.618694

\section{Complete Blood Count Score Model Predicts Inferior Prognosis in Primary Central Nervous System Lymphoma}

\author{
Yuhua Feng ${ }^{1}$, Yiping Liu ${ }^{2}$, Meizuo Zhong ${ }^{2}$ and Leyuan Wang ${ }^{3 *}$ \\ 1 Department of Oncology, The Second Xiangya Hospital, Central South University, Changsha, China, 2 Department of \\ Oncology, Xiangya Hospital, Central South University, Changsha, China, ${ }^{3}$ Department of Pediatrics, Xiangya Hospital, \\ Central South University, Changsha, China
}

Background: Primary central nervous system lymphoma (PCNSL), an aggressive type of non-Hodgkin lymphoma, has a poor prognosis. Currently available prognostic scoring systems are inadequate. We therefore aimed to investigate the predictive values of complete blood counts (CBCs) in PCNSL.

Materials and Methods: The cohort of this retrospective study comprised 73 PCNSL patients. The predictive values of selected CBCs, including neutrophil-to-lymphocyte ratio (NLR), platelet-to-lymphocyte ratio (PLR), systemic immune inflammation index (SII), and systemic inflammation response index (SIRI), were analyzed.

Results: Ages and Memorial Sloan Kettering Cancer Center (MSKCC) scores of PCNSL patients correlated with NLR, PLR, and SII values $(p<0.05)$. Both age and MSKCC scores correlated with inferior progression-free survival (PFS) and overall survival (OS) $(p<0.05)$. High NLR, PLR, SII, and SIRI were significant predictors of shorter PFS and OS $(p<0.05)$. NLR, PLR, SII, and SIRI were integrated to generate a "CBC score" model that accurately stratified PCNSL patients into three risk groups. The median PFS for low-risk, intermediate-risk, and high-risk groups were 24 ((12.458-35.542), 17 (10.626-23.374), and 9 (8.893-19.107) months, respectively $(\rho=0.011)$, and the median OS were 33 (19.175-46.825), 18 (16.368-19.632), and 9 (6.521-11.479) months, respectively $(p=$ 0.008). Multivariate Cox regression model showed that MSKCC score (hazard ratio $(H R)=$ 3.791, $p<0.001)$, PLR (HR = 1.003, $p=0.013)$, and CBC score $(H R=1.873, p=0.011)$ were independent predictors for PFS, whereas MSKCC score $(H R=4.128, p<0.001)$, PLR (HR $=1.003, p=0.005)$, and CBC score $(H R=1.907, p=0.004)$ were independent predictors for OS.

Conclusion: The CBC score model may be a promising predictive system for PCNSL patients.

\section{Keywords: primary central nervous system lymphoma, complete blood count, prognosis, survival, biomarker}




\section{INTRODUCTION}

Primary central nervous system lymphoma (PCNSL), a rare aggressive extranodal subtype of non-Hodgkin lymphoma, is confined to the central nervous system, including the brain, eyes, spine, and pia mater (1). PCNSL represents $1-2 \%$ of all intracranial neoplasms (2) and $4-6 \%$ of all extranodal lymphomas (3). Since 2000, an increasing incidence of PCNSL has been recognized (4). Most PCNSLs are diffuse large B cell lymphoma (DLBCL), rare forms include T-cell lymphoma and Burkitt lymphoma. The prognoses of patients with PCNSL are poor, the 5-year survival rate being 33\%. Without treatment, the disease progresses rapidly, the overall survival (OS) being only 1.5 months $(5,6)$. Identification of accurate prognostic indicators for PCNSL is urgently required.

Two scoring systems are currently used to predict the prognosis of PCNSL patients: those of the International Extranodal Lymphoma Study Group (IELSG) score and Memorial Sloan Kettering Cancer Center (MSKCC) prognostic score. The IELSG scoring system incorporates five variables, namely age, Eastern Cooperative Oncology Group performance score (ECOG PS), lactate dehydrogenase (LDH) level, cerebrospinal fluid (CSF) protein concentration and deep brain involvement (7), whereas the MSKCC scoring system uses only two variables-age and Karnofsky performance status (KPS) (8). In addition to these two scoring systems, $B C L-6$ rearrangement, $6 q 22$ deletion, CXCL13 are reportedly associated with poor prognoses $(9,10)$. With the optimization of PCNSL treatment regimens, the application of molecular targeted therapy and immunotherapy, prognostic score systems for PCNSL need to be further improved.

Previous studies have shown that host immunity and systemic inflammatory responses are closely related to the development and prognosis of cancers $(11,12)$. The complete blood counts (CBCs) such as neutrophil-to-lymphocyte ratio (NLR) and platelet-to-lymphocyte ratio (PLR) have shown their values in predicting the prognosis of cancers. Amounts of evidences have confirmed the values of CBCs in predicting prognoses of gastric cancer (13), breast cancer (14), liver cancer (15) and lymphoma (16). However, little is known about the prognostic value of CBCs in PCNSL.

In this retrospective study, we aimed to investigate the prognostic predicting values of NLR, PLR, systemic immune inflammation index (SII) and systemic inflammation response index (SIRI) in a cohort of 73 PCNSL patients, and evaluate a new CBC score model for predicting prognoses of PCNSL patients, which hasn't been studied before.

\section{PATIENTS AND METHODS}

\section{Patient Selection}

A cohort of 73 patients diagnosed with PCNSL at Xiangya Hospital, Central South University from January 2012 to December 2016 was retrospectively analyzed. The inclusion criterion was that patients were pathologically confirmed
PCNSL. The exclusion criteria were as follows: (1) received chemotherapy, radiotherapy or other anti-tumor therapies before diagnosed, (2) history of autoimmune diseases, (3) history of chronic inflammatory diseases such as inflammatory bowel disease, (4) with uncontrolled active infections or other illnesses.

All 73 PCNSL patients had complete clinical and follow-up data and had been followed-up from the day of diagnosis to June 2020, no patients lost to follow-up. This study was conducted in accordance with the Helsinki Declaration of 1975, revised in 2008 .

\section{Data Collection}

Clinical data including gender, age, KPS, MSKCC score, B symptoms, LDH level, and treatment regimens, were collected. Routine blood results within 1 week before initiation of therapy were also collected. The NLR, PLR, SII, SIRI were calculated as follows: NLR = neutrophil count/lymphocyte count, PLR = platelet count/lymphocyte count, SII $=$ platelet count $\times$ neutrophil count/lymphocyte count, SIRI = neutrophil count $\times$ monocyte count/lymphocyte count. A new CBC score model incorporating these four variables was defined as follows: a score of 1 (low-risk) represents no high expression of CBCs, a score of 2 (intermediate-risk) represents 1-2 high expressions, and a score of 3 (high-risk) represents 3-4 high expressions. Overall survival (OS) was defined as the length of time from the date of diagnosis to the date of death for any cause or to the date of last follow-up; whereas progression free survival (PFS), was defined as the length of time from the date of diagnosis to the date of disease progression or death.

\section{Statistical Analysis}

SPSS statistical software was used for data analysis (version 22.0; SPSS Inc., Chicago, IL, USA). Pearson's $\chi^{2}$ test was used to analyze the relationships between NLR, PLR, SII, SIRI and clinicopathological features of PCNSL patients. Cut-off values of CBCs were calculated by receiver operating characteristic (ROC) curves. Survival curves were calculated according to the Kaplan-Meier method and compared using log-rank test. Multivariate analysis was based on the COX regression model. Two-sided $p$ values $<0.05$ were considered statistical significance.

\section{RESULTS}

\section{Patient Characteristics}

Relevant clinical data of the 73 PCNSL patients are summarized in Table 1. Fifteen of them (20.5\%) were aged $\leq 50$ years and the remaining $58(79.5 \%)>50$ years. $49(67.1 \%)$ were male, and 24 (32.9\%) female. 16 (21.9\%) presented with elevated LDH level, and four (5.5\%) presented with B symptoms. According to MSKCC score, $20.6 \%$ patients were in the "Age $\leq 50$ " group, $35.6 \%$ were in the "Age $>50$ and KPS $\geq 70$ " group, and $43.8 \%$ were in the "Age $>50$ and KPS $<70$ " group. None of the 73 patients received any anti-tumor therapy before diagnosis, and treatment regimens were known for all patients, 18 (24.7\%) had 
TABLE 1 | Clinicopathological characteristics of patients $(n=73)$.

\begin{tabular}{lc}
\hline Characteristics & Number (\%) \\
\hline Age & \\
$\leq 50$ & $15(20.5 \%)$ \\
$>50$ & $58(79.5 \%)$ \\
Gender & \\
Male & $49(67.1 \%)$ \\
Female & $24(32.9 \%)$ \\
LDH level & \\
Normal & $57(78.1 \%)$ \\
Elevated & $16(21.9 \%)$ \\
B symptoms & \\
Present & $4(5.5 \%)$ \\
Absent & $69(94.5 \%)$ \\
MSKCC score & \\
Age $\leq 50$ & $15(20.6 \%)$ \\
Age $>50$ and KPS $\geq 70$ & $26(35.6 \%)$ \\
Age $>50$ and KPS $<70$ & $32(43.8 \%)$ \\
Treatment regimen & \\
Chemo-radiotherapy & $18(24.7 \%)$ \\
Surgery and chemo-radiotherapy & $53(72.6 \%)$ \\
Surgery & $2(2.7 \%)$ \\
\hline
\end{tabular}

undergone chemo-radiotherapy, 53 (72.6\%) surgery and chemoradiotherapy, and two $(2.7 \%)$ surgery alone.

\section{Optimal Cut-Off Values for CBCs and Their Correlations With Clinicopathological Characteristics}

ROC curves were generated to calculate the optimal cut-off values for CBCs. As shown in Figure 1A, the optimal cut-off values for NLR, PLR, SII, and SIRI for PFS were 3.09 (area under curve $($ AUC $)=0.571$, sensitivity $=0.8$, specificity $=0.426), 152.81$ $(\mathrm{AUC}=0.674$, sensitivity $=0.8$, specificity $=0.574), 427.35(\mathrm{AUC}=$ 0.568 , sensitivity $=1$, specificity $=0.25)$, and $1.44(\mathrm{AUC}=0.521$, sensitivity $=0.8$, specificity $=0.574$ ), respectively. As shown in Figure 1B, the optimal cut-off values for OS were 3.09 (AUC $=0.696$, sensitivity $=1$, specificity $=0.435), 307.07(\mathrm{AUC}=0.681$, sensitivity $=$ 0.5 , specificity $=0.928), 514($ AUC $=0.652$, sensitivity $=1$, specificity $=0.362)$, and $1.44($ AUC $=0.605$, sensitivity $=1$, specificity $=0.58)$ for NLR, PLR, SII, and SIRI, respectively.

As for PCNSL patients' clinicopathological characteristics, age and MSKCC score significantly correlated with the expressions of NLR, PLR and SII $(p<0.05)$, whereas patients' gender, $\mathrm{LDH}$ level, B symptoms or treatment regimens had no significant correlation with CBCs $(p>0.05$, Table 2).

\section{Prognostic Factors}

During follow-up, 67 patients presented with disease progression, the median PFS being 13 months, and 59 patients died, the median OS being 13 months. The clinicopathological characteristics that were significantly associated with PFS and OS are shown in Table 3. Age and MSKCC score contributed to unfavorable predictors for PFS and OS ( $p<0.05$, Figure 3), while patients' gender, LDH level, B symptoms or treatment regimens had no significant correlation with prognosis $(p>0.05)$.

Kaplan-Meier survival curves for PFS and OS with respect to CBCs are shown in Figure 2 and Table 4. As shown in Figures 2A-D, high NLR $(p=0.01)$, high PLR $(p<0.001)$, high SII $(p=$ $0.012)$ and high SIRI $(p=0.024)$ were predictors of shorter PFS. As shown in Figures 2E-H, high NLR $(p=0.008)$, high PLR ( $p=$ $0.002)$, high SII $(p=0.01)$ and high SIRI $(p=0.037)$ were predictors of shorter OS.
A

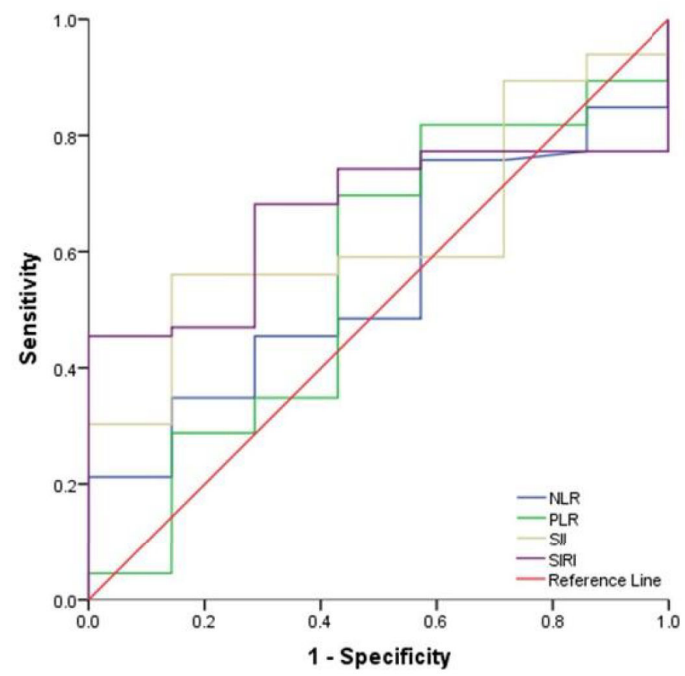

B

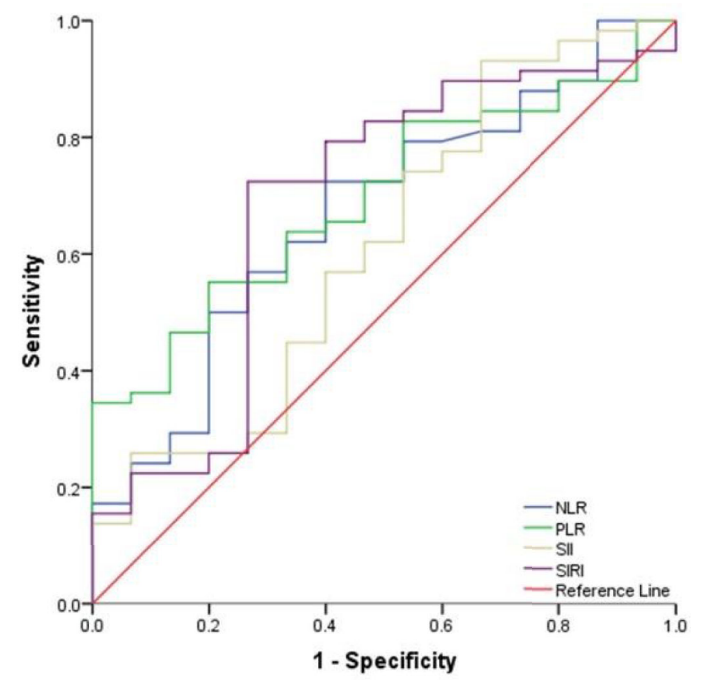

FIGURE 1 | The cut-off values for CBCs. (A) ROC curves analysis for optimal cut-off values of NLR, PLR, SII and SIRI for PFS. (B) Roc curves analysis for optimal cut-off values of NLR, PLR, SII and SIRI for OS. 


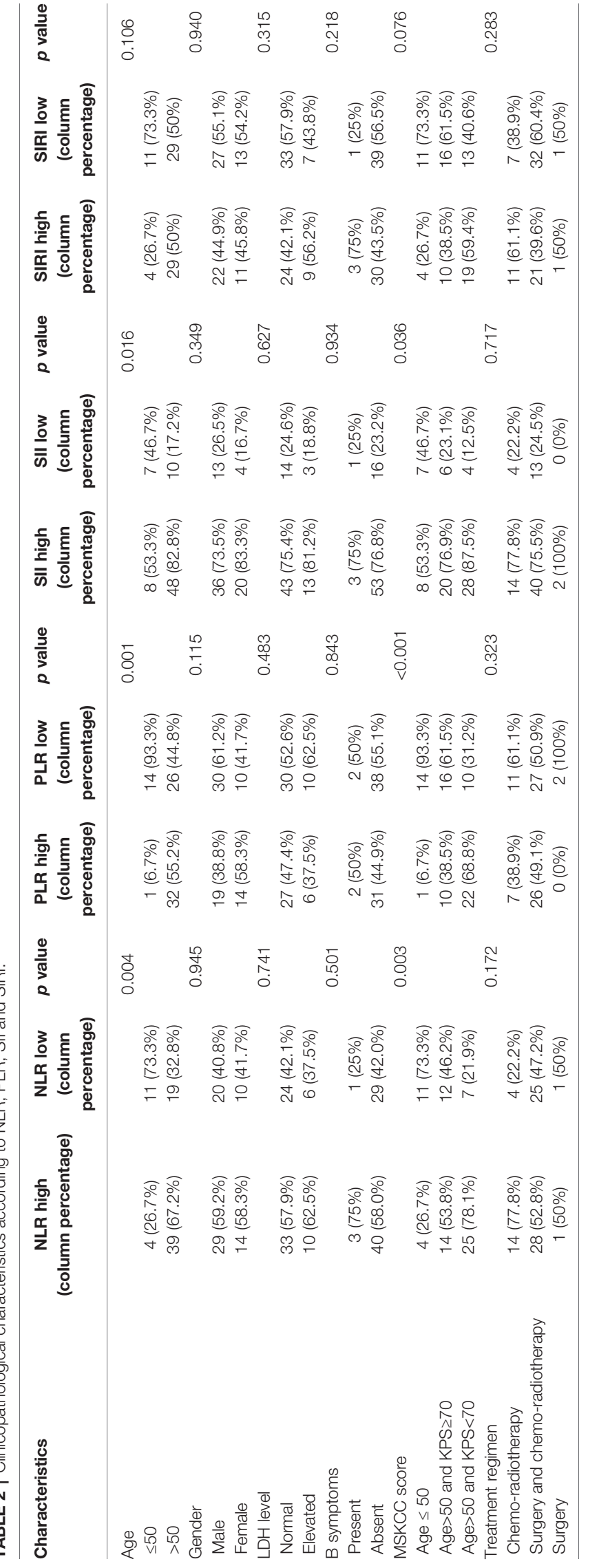

TABLE 3 | Association between clinicopathological characteristics and prognosis.

\begin{tabular}{|c|c|c|c|c|}
\hline Characteristics & $\begin{array}{c}\text { Median PFS } \\
\text { (months, 95\% Cl) }\end{array}$ & $\begin{array}{c}p \\
\text { value }\end{array}$ & $\begin{array}{c}\text { Median OS } \\
\text { (months, } 95 \% \mathrm{Cl} \text { ) }\end{array}$ & $\begin{array}{c}p \\
\text { value }\end{array}$ \\
\hline Age & & $<0.001$ & & $<0.001$ \\
\hline$\leq 50$ & 51 (41.963-60.037) & & 70 (52.903-87.097) & \\
\hline$>50$ & 11 (8.845-13.155) & & 11 (8.514-13.486) & \\
\hline Gender & & 0.527 & & 0.807 \\
\hline Male & $14(5.667-22.333)$ & & 15 (9.216-20.784) & \\
\hline Female & 14 (5.784-22.216) & & $18(11.060-24.940)$ & \\
\hline LDH level & & 0.224 & & 0.206 \\
\hline Normal & $11(6.883-15.117)$ & & $12(6.858-17.142)$ & \\
\hline Elevated & $21(7.120-34.880)$ & & $22(8.428-35.572)$ & \\
\hline B symptoms & & 0.494 & & 0.399 \\
\hline Present & 19 (not applicable) & & $21(7.280-34.720)$ & \\
\hline Absent & $11(7.502-14.498)$ & & $14(9.138-18.862)$ & \\
\hline MSKCC score & & $<0.001$ & & $<0.001$ \\
\hline Age $\leq 50$ & 51 (41.963-60.037) & & 70 (52.903-87.097) & \\
\hline $\begin{array}{l}\text { Age }>50 \text { and } \\
\text { KPS } \geq 70\end{array}$ & $19(14.314-23.686)$ & & 21 (9.687-32.313) & \\
\hline $\begin{array}{l}\text { Age }>50 \text { and } \\
\mathrm{KPS}<70\end{array}$ & 7 (4.714-9.286) & & 7 (4.228-9.772) & \\
\hline Treatment regimen & & 0.932 & & 0.902 \\
\hline $\begin{array}{l}\text { Chemo- } \\
\text { radiotherapy }\end{array}$ & $14(4.429-23.571)$ & & $12(5.763-18.237)$ & \\
\hline $\begin{array}{l}\text { Surgery and } \\
\text { chemo- } \\
\text { radiotherapy }\end{array}$ & $14(8.064-19.936)$ & & $16(10.382-21.618)$ & \\
\hline Surgery & 1 (not applicable)* & & 1 (not applicable) $^{*}$ & \\
\hline
\end{tabular}

*Sample size was not enough to calculate $95 \% \mathrm{Cl}$ of survival time.

\section{The Predictive Value of CBC Score Model for Prognosis}

Given the predictive value of NLR, PLR, SII and SIRI for PCNSL patients' prognoses, a new CBC score model that incorporated these four variables was devised and its value in predicting prognosis evaluated. As shown in Figure 4 and Table 4, PCNSL patients were divided into three risk groups. The median PFS for low-risk, intermediate-risk, and high-risk groups were 24 ((12.458-35.542), 17 (10.626-23.374), and 9 (8.893-19.107) months, respectively $(p=0.011)$, and the median OS were 33(19.175-46.825), 18 (16.368-19.632), and 9 (6.521-11.479) months, respectively $(p=0.008)$. Multivariate Cox regression analysis, including age, MSKCC score, NLR, PLR, SII, SIRI, CBC score showed that MSKCC score (hazard ratio $(\mathrm{HR})=3.791, p<0.001), \mathrm{PLR}(\mathrm{HR}=1.003, p=0.013)$, and CBC score model ( $\mathrm{HR}=1.873, p=0.011)$ were independent predictors for PFS, and MSKCC score $(\mathrm{HR}=4.128, p<0.001)$, PLR $(\mathrm{HR}=$ $1.003, p=0.005)$, and CBC score model $(\mathrm{HR}=1.907, p=0.004)$ were independent predictors for OS (Table 5).

\section{DISCUSSION}

PCNSL is an aggressive type of non-Hodgkin lymphoma. Because it is rare, no standard treatment regimen has been established and its prognosis remains poor. In the present study, we evaluated the prognosis values of CBCs, including NLR, PLR, SII, and SIRI, in 73 PCNSL patients, and built a newly-defined CBC score model to stratify these patients into three risk groups. We found high NLR, PLR, SII, and SIRI to be 


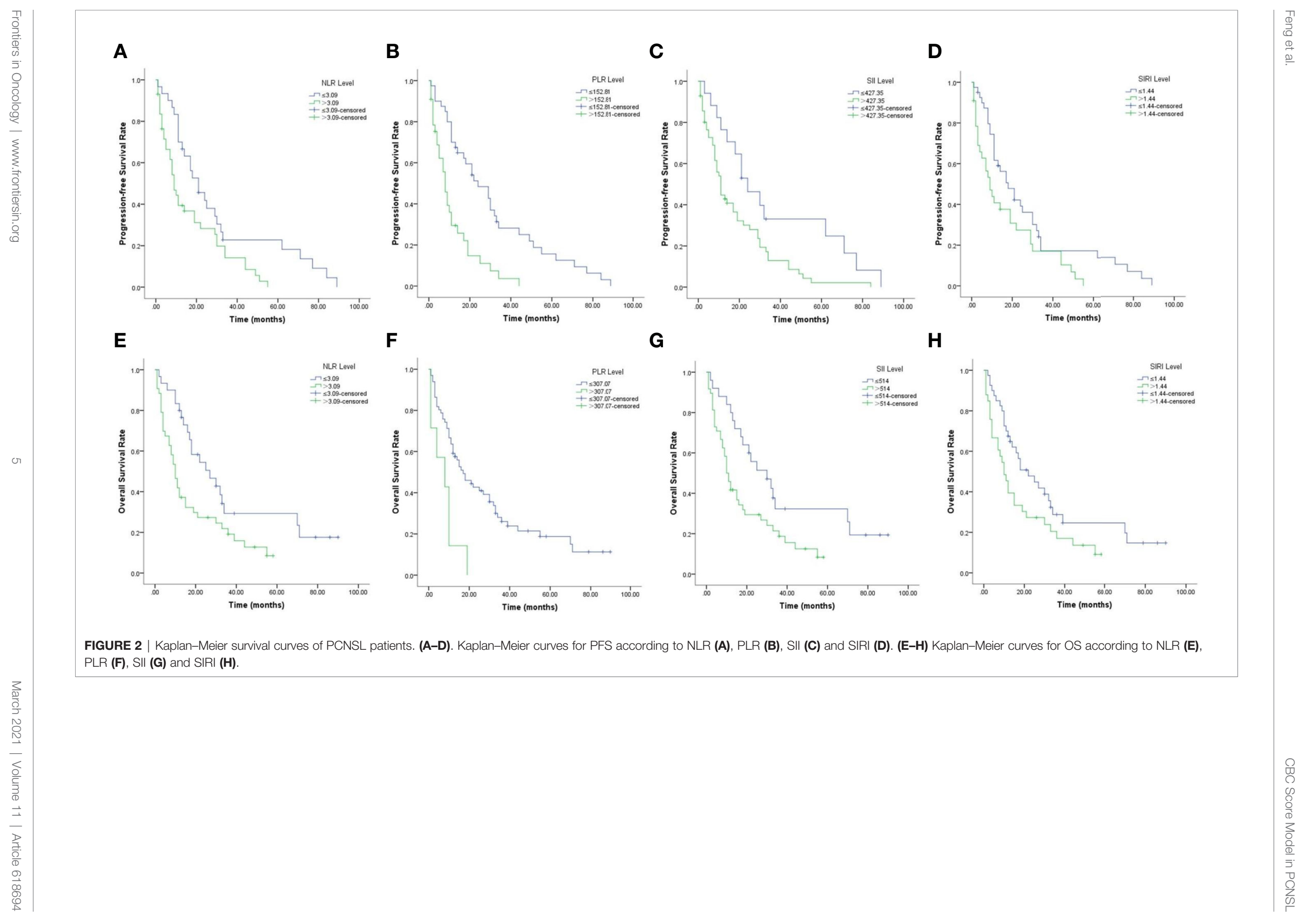


A

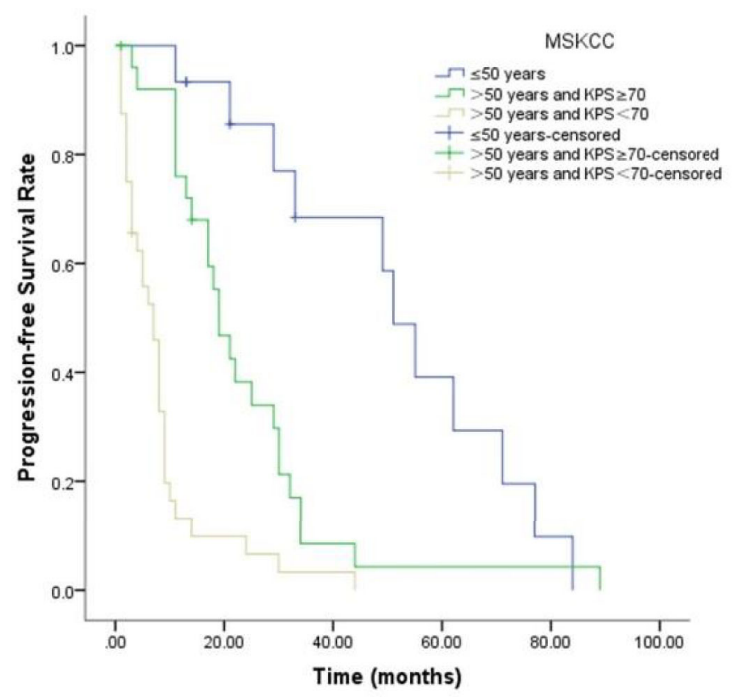

B

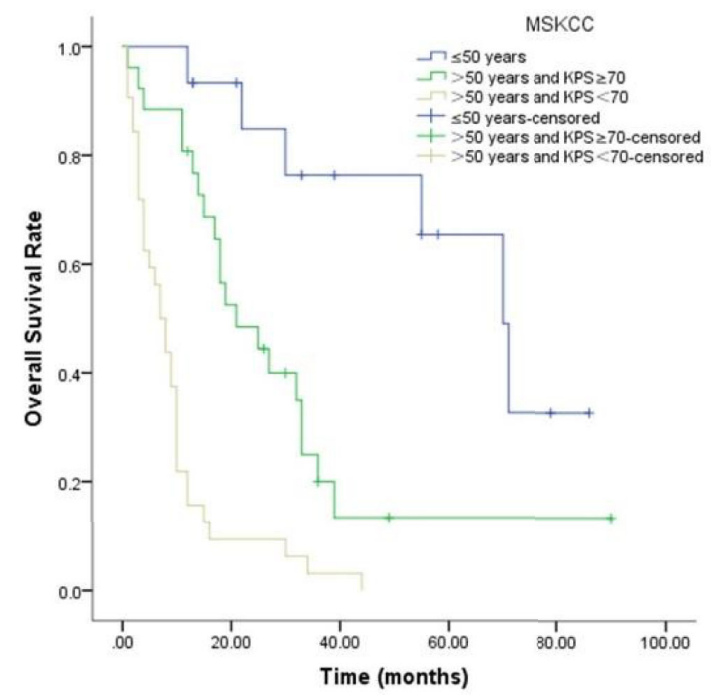

FIGURE 3 | Prognostic MSKCC scores in PCNSL patients. (A) Results for PFS. (B) Results for OS.

TABLE 4 | Univariate analysis of CBCs associated with PFS and OS.

\begin{tabular}{lcccc}
\hline CBCs & $\begin{array}{c}\text { Median PFS } \\
\text { (months, 95\% Cl) }\end{array}$ & p value & $\begin{array}{c}\text { Median OS } \\
\text { (months, 95\% Cl) }\end{array}$ & p value \\
\hline $\begin{array}{l}\text { NLR } \\
\text { High }\end{array}$ & $9(6.342-11.658)$ & & $10(7.253-12.747)$ & 0.008 \\
Low & $21(12.160-29.840)$ & & $27(12.564-41.436)$ & \\
PLR & & $<0.001$ & & 0.002 \\
High & $8(5.856-10.144)$ & & $8(0.510-18.256)$ & \\
Low & $24(13.586-34.414)$ & & $17(9.913-20.087)$ & \\
SII & & 0.012 & & 0.01 \\
High & $11(8.627-13.373)$ & & $10(7.454-12.536)$ & \\
Low & $24(12.458-35.542)$ & & $30(15.060-44.940)$ & \\
SIRI & & 0.024 & $10(6.249-13.751)$ & \\
High & $9(4.862-13.138)$ & & $22(10.952-33.048)$ & \\
Low & $18(10.060-25.940)$ & & & \\
CBC score & & & & \\
1 & $24(12.458-35.542)$ & 0.011 & $33(19.175-46.825)$ & 0.008 \\
2 & $17(10.626-23.374)$ & & $18(16.368-19.632)$ & \\
3 & $9(8.893-19.107)$ & & $9(6.521-11.479)$ & \\
\hline
\end{tabular}

closely associated with inferior PFS and OS, and that our CBC score model could be used to stratify patients with PCNSL into risk categories and predict their prognoses.

The systemic inflammatory responses are reported to be closely related to the development of various cancers, and play a key role in predicting prognosis (17). The CBCs consist of platelets, neutrophils, lymphocytes, monocytes, etc., and there is now increasing evidences that $\mathrm{CBCs}$ can be used to predict outcomes of cancers. The combined CBCs of the above variables, such as NLR (18), PLR (19, 20), SII (21, 22), and SIRI (23), have been reported to be useful prognostic indicators in some cancers. Furthermore, because blood cells are routinely counted, related data is easy to access, which makes CBCs promising candidates for prognostic indicators in cancers.
In the present study, we calculated optimal cut-off values for NLR, PLR, SII, and SIRI, and classified them into high- and lowlevel groups accordingly. In the present cohort, age and MSKCC score of 73 PCNSL patients were significantly associated with the expressions of NLR, PLR, and SII $(p<0.05)$, while gender, LDH level, B symptoms or treatment regimens had no significant correlation with CBCs. In PCNSL, MSKCC score system is one of the most commonly used predicting systems in PCNSL, having been shown to enable stratification of these patients into three risk groups on the basis of age and KPS: age $\leq 50$ years, age $>50$ years and KPS $\geq 70$, or age $>50$ years and KPS $<70$. MSKCC score system is reported to be closely related to patients' OS (8). Our findings showed age and MSKCC score, which were prognosis predictors for PCNSL, were associated with CBCs, that indicates CBCs may be related to the prognosis in PCNSL. Further analysis showed that age and MSKCC score contributed to unfavorable predictors for PFS and OS, that result was consistent with the previous study.

We also found that PCNSL patients with high NLR, PLR, SII, and SIRI had inferior PFS and OS compared with those with low NLR, PLR, SII, and SIRI. The prognostic values of NLR, PLR, SII and SIRI have been reported in many types of cancers. Xia et al. (24) analyzed the prognostic value of NLR in a cohort of 359 patients with osteosarcoma, and found that patients with NLR $\geq 3.43$ had inferior 5-year OS and PFS, and that NLR was an independent prognostic indicator for prognosis. In PCNSL, a previous study reported that patients with NLR $\geq 2.0$ had both worse 3-year OS (42.5\% vs. 71.2\%; $p=0.031$ ) and 3-year PFS (37.3\% vs. $60.1 \% ; p=0.028$ ) (25). Suzuki et al. reported that comparing with lower NLR and PLR, limited-stage small-cell lung cancer patients with higher NLR and PLR had worse median and 2-year OS (NLR: 14.9 vs. 17.8 months, $29 \%$ vs. $31 \%$; $p=0.026$; PLR: 14.8 vs. 18.9 months, $24 \%$ vs. $37 \%$; $p=0.009$ ) (26). A retrospective analysis of 1,383 patients with colorectal cancer showed that OS 
A

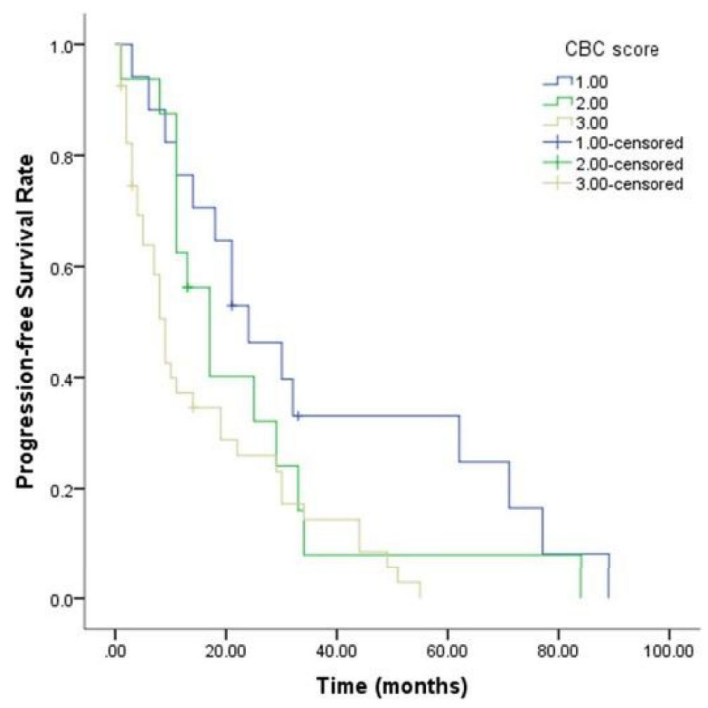

B

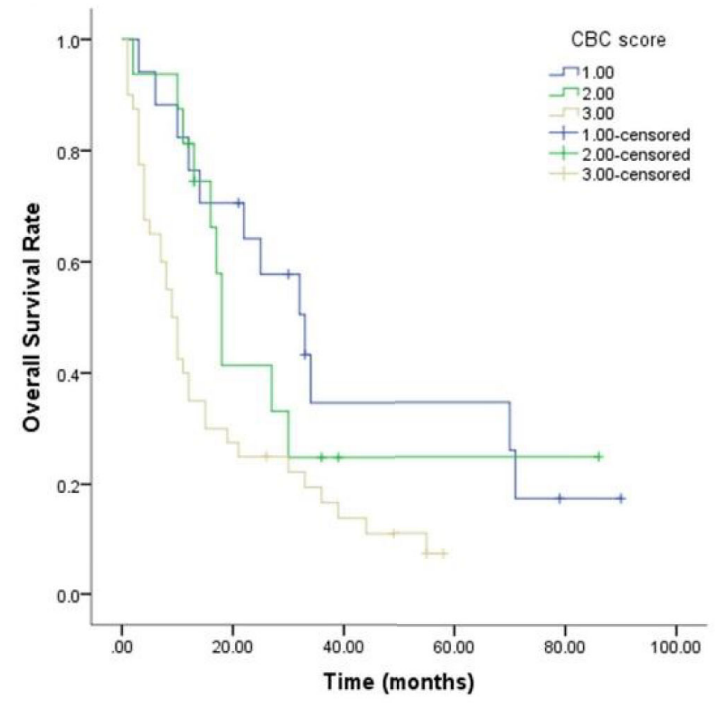

FIGURE 4 | Prognostic CBC score model in PCNSL patients. (A) Results for PFS. (B) Results for OS.

TABLE 5 | Multivariate analysis of prognostic factors affecting PFS and OS.

\begin{tabular}{lccccccc}
\hline \multirow{2}{*}{ Parameters } & \multicolumn{4}{c}{ PFS } & & \multicolumn{3}{c}{ OS } \\
\cline { 2 - 3 } \cline { 7 - 8 } & HR & $\mathbf{9 5 \%} \mathbf{~ C l}$ & $\boldsymbol{p}$ value & HR & $\mathbf{9 5 \%} \mathbf{~ C l}$ & $\boldsymbol{p}$ value \\
\hline Age & 0.69 & $0.220-2.166$ & 0.525 & & 0.82 & $0.218-3.08$ & 0.769 \\
MSKCC score & 3.791 & $2.041-7.042$ & $<0.001$ & & 4.128 & $2.192-7.773$ & $<0.001$ \\
NLR & 1.142 & $0.917-1.423$ & 0.235 & & 1.212 & $0.969-1.517$ & 0.093 \\
PLR & 1.003 & $1.001-1.005$ & 0.013 & & 1.003 & $1.001-1.006$ & 0.005 \\
SII & 1 & $0.999-1.001$ & 0.637 & & 0.999 & $0.998-1$ & 0.202 \\
SIRI & 0.902 & $0.74-1.101$ & 0.311 & & 0.959 & $0.761-1.209$ & 0.724 \\
CBC score & 1.873 & $1.563-2.301$ & 0.011 & & 1.907 & $1.612-2.451$ & 0.004 \\
\hline
\end{tabular}

and disease-free survival (DFS) were better in patients with low NLR, PLR, and SII, and that SII was an independent predictor for OS and DFS (27). Qi et al. reported that patients with advanced pancreatic cancer, who had a SIRI $\geq 1.8$ had shorter time to progression and shorter OS than those with lower SIRI, and multivariate analysis confirmed that SIRI was an independent prognostic factor for both TTP and OS (28). The above results were consistent with ours, indicating that CBCs including NLR, PLR, SII, and SIRI, may be prognostic factors for PCNSL patients.

We defined a new CBC score model and evaluated its prognostic value in PCNSL patients. We found that the median PFS for low-risk, intermediate-risk and high-risk groups were 24 ((12.458-35.542), 17 (10.626-23.374), and 9 (8.893-19.107) months, respectively $(p=0.011)$, and the median OS were 33 (19.175-46.825), 18 (16.368-19.632), and 9 (6.521-11.479) months, respectively $(p=0.008)$, indicating that patients in the high-risk group had a significantly inferior prognosis. In a previous retrospective study of adults with $\mathrm{T}$ lymphoblastic lymphoma, a similar CBC score model consisting of lymphocyte-monocyte ratio, NLR, and PLR was evaluated, and it was found that the median PFS for the low-risk, intermediate-risk, and high-risk groups was not reached, 16 months, and 7 months, respectively $(p=0.004)$, whereas the median OS for these three groups was not reached, 46 months, and 20 months, respectively $(p=0.007)$ (29). These findings were consistent with ours, indicating that $\mathrm{CBC}$ score model is valuable in predicting PCNSL patient's prognosis.

This study had several limitations. First, it was a small, retrospective, single center study. Second, the correlation between specific treatment regimens or treatment responses with prognosis was not validated. Third, the CBCs biomarkers could be influenced by various unidentified factors, such as immune status, which could bring bias to results. Further studies are needed to confirm the role of CBCs in PCNSL patients.

In summary, in the present study we showed that CBCs including NLR, PLR, SII, and SIRI can serve as prognostic indicators in PCNSL patients, and that our newly-defined CBC score model may be a promising predictive score system for PCNSL patients.

\section{DATA AVAILABILITY STATEMENT}

The original contributions presented in the study are included in the article/supplementary material. Further inquiries can be directed to the corresponding author.

\section{ETHICS STATEMENT}

The studies involving human participants were reviewed and approved by the Ethics Committee of Xiangya Hospital of Central South University. The patients/participants provided their written informed consent to participate in this study. 


\section{AUTHOR CONTRIBUTIONS}

LW designed the research study and collected the clinical data. YF performed the research and drafted the manuscript. YL and $\mathrm{MZ}$ participated in the literature search and analyzed the data. All authors contributed to the article and approved the submitted version.

\section{REFERENCES}

1. Swerdlow SH, Campo E, Pileri SA, Harris NL, Stein H, Siebert R, et al. The 2016 revision of the World Health Organization classification of lymphoid neoplasms. Blood (2016) 127(20):2375-90. doi: 10.1182/blood-2016-01643569

2. Ostrom QT, Patil N, Cioffi G, Waite K, Kruchko C, Barnholtz-Sloan JS. CBTRUS Statistical Report: Primary Brain and Other Central Nervous System Tumors Diagnosed in the United States in 2013-2017. Neuro Oncol (2020) 22 (12 Suppl 2):iv1-iv96. doi: 10.1093/neuonc/noaa200

3. Grommes C, Deangelis LM. Primary CNS Lymphoma. J Clin Oncol (2017) 35 (21):2410-8. doi: 10.1200/JCO.2017.72.7602

4. Mendez JS, Ostrom QT, Gittleman H, Kruchko C, Deangelis LM, BarnholtzSloan JS, et al. The elderly left behind-changes in survival trends of primary central nervous system lymphoma over the past 4 decades. Neuro Oncol (2018) 20(5):687-94. doi: 10.1093/neuonc/nox187

5. Shiels MS, Pfeiffer RM, Besson C, Clarke CA, Morton LM, Nogueira L, et al. Trends in primary central nervous system lymphoma incidence and survival in the U.S. Br J Haematol (2016) 174(3):417-24. doi: 10.1111/bjh.14073

6. Panageas KS, Elkin EB, Deangelis LM, Ben-Porat L, Abrey LE. Trends in survival from primary central nervous system lymphoma, 1975-1999: a population-based analysis. Cancer (2005) 104(11):2466-72. doi: 10.1002/ cncr.21481

7. Ferreri AJ, Blay JY, Reni M, Pasini F, Spina M, Ambrosetti A, et al. Prognostic scoring system for primary CNS lymphomas: the International Extranodal Lymphoma Study Group experience. J Clin Oncol (2003) 21(2):266-72. doi: 10.1200/JCO.2003.09.139

8. Abrey LE, Ben-Porat L, Panageas KS, Yahalom J, Berkey B, Curran W, et al. Primary central nervous system lymphoma: the Memorial Sloan-Kettering Cancer Center prognostic model. J Clin Oncol (2006) 24(36):5711-5. doi: 10.1200/JCO.2006.08.2941

9. Cady FM, O'neill BP, Law ME, Decker PA, Kurtz DM, Giannini C, et al. Del (6)(q22) and BCL6 rearrangements in primary CNS lymphoma are indicators of an aggressive clinical course. J Clin Oncol (2008) 26(29):4814-9. doi: 10.1200/JCO.2008.16.1455

10. Rubenstein JL, Wong VS, Kadoch C, Gao HX, Barajas R, Chen L, et al. CXCL13 plus interleukin 10 is highly specific for the diagnosis of CNS lymphoma. Blood (2013) 121(23):4740-8. doi: 10.1182/blood-2013-01-476333

11. Savas P, Salgado R, Denkert C, Sotiriou C, Darcy PK, Smyth MJ, et al. Clinical relevance of host immunity in breast cancer: from TILs to the clinic. Nat Rev Clin Oncol (2016) 13(4):228-41. doi: 10.1038/nrclinonc.2015.215

12. Feliciano EMC, Kroenke CH, Meyerhardt JA, Prado CM, Bradshaw PT, Kwan ML, et al. Association of Systemic Inflammation and Sarcopenia With Survival in Nonmetastatic Colorectal Cancer: Results From the C SCANS Study. JAMA Oncol (2017) 3(12):e172319. doi: 10.1001/jamaoncol.2017.2319

13. Sun J, Chen X, Gao P, Song Y, Huang X, Yang Y, et al. Can the Neutrophil to Lymphocyte Ratio Be Used to Determine Gastric Cancer Treatment Outcomes? A Systematic Review and Meta-Analysis. Dis Markers (2016) 2016:7862469. doi: 10.1155/2016/7862469

14. Ethier JL, Desautels D, Templeton A, Shah PS, Amir E. Prognostic role of neutrophil-to-lymphocyte ratio in breast cancer: a systematic review and meta-analysis. Breast Cancer Res (2017) 19(1):2. doi: 10.1186/s13058-0160794-1

15. Zheng J, Cai J, Li H, Zeng K, He L, Fu H, et al. Neutrophil to Lymphocyte Ratio and Platelet to Lymphocyte Ratio as Prognostic Predictors for Hepatocellular Carcinoma Patients with Various Treatments: a MetaAnalysis and Systematic Review. Cell Physiol Biochem (2017) 44(3):967-81. doi: $10.1159 / 000485396$

\section{ACKNOWLEDGMENTS}

The authors thank the Department of Oncology, The Second Xiangya Hospital of Central South University, Department of Oncology, Xiangya Hospital of Central South University and Department of Pediatrics, Xiangya Hospital of Central South University for technical support.

16. Annibali O, Hohaus S, Marchesi F, Cantonetti M, Di Rocco A, Tomarchio V, et al. The neutrophil/lymphocyte ratio $\geq 3.5$ is a prognostic marker in diffuse large B-cell lymphoma: a retrospective analysis from the database of the Italian regional network 'Rete Ematologica del Lazio per i Linfomi' (RELLI). Leuk Lymphoma (2019) 60:(14):3386-94. doi: 10.1080/10428194.2019. 1633628

17. Dolan RD, Mcsorley ST, Horgan PG, Laird B, Mcmillan DC. The role of the systemic inflammatory response in predicting outcomes in patients with advanced inoperable cancer: Systematic review and meta-analysis. Crit Rev Oncol Hematol (2017) 116:134-46. doi: 10.1016/j.critrevonc.2017.06.002

18. Miyamoto R, Inagawa S, Sano N, Tadano S, Adachi S, Yamamoto M. The neutrophil-to-lymphocyte ratio (NLR) predicts short-term and long-term outcomes in gastric cancer patients. Eur J Surg Oncol (2018) 44(5):607-12. doi: 10.1016/j.ejso.2018.02.003

19. Bilen MA, Martini DJ, Liu Y, Lewis C, Collins HH, Shabto JM, et al. The prognostic and predictive impact of inflammatory biomarkers in patients who have advanced-stage cancer treated with immunotherapy. Cancer (2019) 125 (1):127-34. doi: $10.1002 / \mathrm{cncr} .31778$

20. Diem S, Schmid S, Krapf M, Flatz L, Born D, Jochum W, et al. Neutrophil-toLymphocyte ratio (NLR) and Platelet-to-Lymphocyte ratio (PLR) as prognostic markers in patients with non-small cell lung cancer (NSCLC) treated with nivolumab. Lung Cancer (2017) 111:176-81. doi: 10.1016/ j.lungcan.2017.07.024

21. Xie QK, Chen $\mathrm{P}, \mathrm{Hu}$ WM, Sun $\mathrm{P}, \mathrm{He}$ WZ, Jiang $\mathrm{C}$, et al. The systemic immune-inflammation index is an independent predictor of survival for metastatic colorectal cancer and its association with the lymphocytic response to the tumor. J Transl Med (2018) 16(1):273. doi: 10.1186/s12967018-1638-9

22. Wang Y, Li Y, Chen P, Xu W, Wu Y, Che G. Prognostic value of the pretreatment systemic immune-inflammation index (SII) in patients with non-small cell lung cancer: a meta-analysis. Ann Trans Med (2019) 7(18):433. doi: 10.21037/atm.2019.08.116

23. Wang L, Zhou Y, Xia S, Lu L, Dai T, Li A, et al. Prognostic value of the systemic inflammation response index (SIRI) before and after surgery in operable breast cancer patients. Cancer Biomark (2020) 28(4):537-47. doi: 10.3233/CBM-201682

24. Xia WK, Liu ZL, Shen D, Lin QF, Su J, Mao WD. Prognostic performance of pre-treatment NLR and PLR in patients suffering from osteosarcoma. World J Surg Oncol (2016) 14:127. doi: 10.1186/s12957-016-0889-2

25. Jung J, Lee H, Yun T, Lee E, Moon H, Joo J, et al. Prognostic role of the neutrophil-to-lymphocyte ratio in patients with primary central nervous system lymphoma. Oncotarget (2017) 8(43):74975-86. doi: 10.18632/ oncotarget. 20480

26. Suzuki R, Wei X, Allen PK, Cox JD, Komaki R, Lin SH. Prognostic Significance of Total Lymphocyte Count, Neutrophil-to-lymphocyte Ratio, and Platelet-to-lymphocyte Ratio in Limited-stage Small-cell Lung Cancer. Clin Lung Cancer (2019) 20(2):117-23. doi: 10.1016/j.cllc.2018. 11.013

27. Chen JH, Zhai ET, Yuan YJ, Wu KM, Xu JB, Peng JJ, et al. Systemic immuneinflammation index for predicting prognosis of colorectal cancer. World $J$ Gastroenterol (2017) 23(34):6261-72. doi: 10.3748/wjg.v23.i34.6261

28. Qi Q, Zhuang L, Shen Y, Geng Y, Yu S, Chen H, et al. A novel systemic inflammation response index (SIRI) for predicting the survival of patients with pancreatic cancer after chemotherapy. Cancer (2016) 122(14):2158-67. doi: 10.1002/cncr.30057

29. Feng X, Li L, Wu J, Zhang L, Sun Z, Li X, et al. Complete Blood Count Score Model Integrating Reduced Lymphocyte-Monocyte Ratio, Elevated Neutrophil-Lymphocyte Ratio, and Elevated Platelet-Lymphocyte Ratio 
Predicts Inferior Clinical Outcomes in Adult T-Lymphoblastic Lymphoma. Oncologist (2019) 24(11):e1123-31. doi: 10.1634/theoncologist.2018-0789

Conflict of Interest: The authors declare that the research was conducted in the absence of any commercial or financial relationships that could be construed as a potential conflict of interest.
Copyright $\odot 2021$ Feng, Liu, Zhong and Wang. This is an open-access article distributed under the terms of the Creative Commons Attribution License (CC BY). The use, distribution or reproduction in other forums is permitted, provided the original author(s) and the copyright owner(s) are credited and that the original publication in this journal is cited, in accordance with accepted academic practice. No use, distribution or reproduction is permitted which does not comply with these terms. 